\title{
Comment on the article Genetic contribution to all cancers: the first demonstration using the model of breast cancers from Poland stratified by age at diagnosis and tumour pathology by Lubinski et al., Breast Cancer Res Treat 2008 Apr 15
}

\author{
Arvids Irmejs, Edvins Miklaševics, Ilze Štrumfa, Janis Gardovskis \\ Riga Stradins University, Hereditary Cancer Institute, Riga, Latvia
}

Corresponding author: Hereditary Cancer Institute, Riga Stradins University, Dzirciema Street 16, LV 1007, Riga, Latvia, phone: +37 170699 73,fax: +37 170699 73, e-mail: hci@stradini.lv

The study has set an important and complicated goal - to verify the hypothesis that genetic polymorphisms are associated with a predisposition to all malignancies.

The article is attractive because of the thorough design, involving a large number of cases and carefully matched controls. The approach by dividing the whole group of breast cancers into 20 subgroups by histological type, grade, and receptor is original and interesting. As it is applied with great care, the data indeed provide novel information about the genetic basis of breast cancer genesis and possibly provide evidence that different types of breast cancer might have different pathogenesis. These data could be helpful in further basic research on breast tumorigenesis, highlighting genetic characteristics for each type of breast cancer. The authors have also shown that heterogeneity of breast cancer at the genetic level is as diverse as the morphological picture, a fact that has to be taken into account in future research design.

Comparison of frequencies of polymorphisms in several genes between two groups (cancer patients and controls) in an otherwise homogeneous population leads the authors to several important, and fatalistic, statements: - genetic components play a role in all cancers,

- environmental factors act effectively only on predisposed persons.

Though the first conclusion seems to be acceptable to a large extent intuitively, the data provided by the authors do not strengthen this belief, at least for us. Thus, $90.6 \%$ of cancer patients harboured at least one of the studied markers. But the same is true for more than $80 \%$ of the control group. Why in one group are these low and medium penetrance genes considered as factors leading to development of breast cancer (or any cancer in general) and ignored in the other group? What makes a difference between two carriers of e.g. CHK2 mutation? Are there other, as yet unclear, genetic components which increase the significance of this mutation? Or maybe some environmental factors? The authors have taken great pains to stratify the two groups but have ignored any lifestyle aspects.
There is no doubt that mutation carriers in genes involved in DNA repair can be affected by a hostile environment more severely and accumulate a greater number of somatic mutations. It is estimated that the TP53 gene is mutated in about $50 \%$ of cancers [1]. Should we call such cancer sporadic, or is it genetically predetermined now? Do the authors believe that persons not possessing the listed (and some other) markers could smoke, be obese and enjoy mutagens without being punished?

Taking into account the high rate of genetic polymorphisms in the population, the aim of the study - to verify the hypothesis that genetic polymorphisms are associated with a predisposition to all malignancies - is hardly achieved as the relative risk seems to be small and thus other, possibly environmental factors could easily have a greater impact on the outcome in terms of tumour development. The results might reflect, in fact, the genetic variability within the population.

According to the results, $9 \%$ of breast cancer cases did not show any marker and $16.6 \%$ (162) of control group women were free of any marker tested. The main clinical consequence of the study in our opinion could be the possibility to release "marker negative" women from an unnecessary mammographic screening programme. But to receive the ultimate verification of the hypothesis, it will be necessary to perform a follow-up study on this group of women and to wait some $30-40$ years. Besides, there is a great probability that during the next years several more cancer predisposing candidate genes will be discovered and it could easily lead to the situation that at least one marker will be found in $100 \%$ of cases and controls. If so, this study approach could be considered as an important contribution to overall understanding about tumorigenesis, but it is difficult to predict its application in clinical practice in the near future.

\section{References}

1. Vogelstein B, Lane D, Levine AJ. Surfing the p53 network. Nature 2000; 408: 307-310. 